TIP Periodica Polytechnica

Transportation Engineering

42(1), pp. 19-25, 2014

DOI: $10.3311 /$ PPtr. 7280

http://www.pp.bme.hu/tr/article/view/7280

Creative Commons Attribution (i)

RESEARCH ARTICLE

\section{Integrated vehicle control of in-wheel electric vehicle}

\author{
András Mihály / Balázs Németh / Péter Gáspár
}

ReCeived 4 November 2013; ACcepted 6 January 2014

\begin{abstract}
The paper presents a control method for in-wheel electric vehicles, integrating several vehicle components such as steering, braking and propulsion system. The aim is to design a cruise control system with trajectory tracking ability. First a control oriented vehicle model is introduced and the trajectory and velocity tracking problem is formulated. Next the robust LPV (Linear Parameter Varying) trajectory tracking cruise control design is introduced. In the implementation of the integrated control system, the limitations of the actuators has to be considered as well as the road surface adhesion. The operation of the proposed system is demonstrated in CarSim simulation environment with maneuvering the vehicle along a racetrack with altering speed limits.
\end{abstract}

\author{
Keywords \\ in-wheel vehicle $\cdot$ integrated control $\cdot$ trajectory tracking
}

\section{András Mihály}

Department of Control for Transportation and Vehicle Systems,

Budapest University of Technology and Economics,

Stoczek J. u. 2., H-1111 Budapest, Hungary

e-mail: mihaly.andras@mail.bme.hu

\section{Balázs Németh}

Institute for Computer Science and Control, Hungarian Academy of Sciences,

Kende u. 13-17., H-1111 Budapest, Hungary

e-mail: nemeth.balazs@sztaki.mta.hu

Péter Gáspár

Institute for Computer Science and Control, Hungarian Academy of Sciences, Kende u. 13-17., H-1111 Budapest, Hungary

e-mail: gaspar.peter@sztaki.mta.hu

\section{Introduction}

Integrated vehicle control methods has been researched by several authors recently (Dugard et al., Scacchioli et al., Yu et al., Németh et al.). The main advantage of integrated control is the coordinated control of vehicle subsystems with respect to the coupling of vehicle dynamics. By integrated control of steering, braking, suspension the conflicts among these subsystems can be avoided by the supervision of a central controller. The aim of the integrated control can differ. Roll motion control has been presented by several authors (Kim, Hori et $a l$.), where the control goal is to decrease the roll motion of the vehicle in order to avoid dangerous rollover. One of the most important task in vehicle control is to preserve yaw stability during cornering in order to avoid skidding of the vehicle. In the research papers and industrial practice differential braking is the most popular way to realize yaw stability of the vehicle, although this can be achieved by active torque distribution (Zhang et al.) and active steering (Anwar et al.) as well. Integrated control that cooperates four wheel steering and yaw moment control to improve the vehicle handling performance and stability has also been researched (Jianyong et al., Wang et al.). Active steering and suspension controllers can also be integrated to improve yaw and roll stability (Baslamisli et al., Mastinu et al., Gáspár et al.). In the paper, the vehicle is controlled with the propulsion of in-wheel electric motors acting on all four wheels, active steering of the front wheels and differential braking of the rear wheels. For the controller design a single track bicycle model of the vehicle is used.

The controller design is evaluated using LPV framework. This paper is organized as follows: in Section 2 the nonlinear control-oriented vehicle model is formulated. Section 3 proposes the design of integrated vehicle control based on robust LPV framework. Section 4 deals with the distribution of the wheel forces and the yaw torque within the propulsion, the steering and the brake system. The trajectory and velocity tracking control method is validated by simulation in Section 5 . Finally, some concluding remarks are given in Section 6. 


\section{Control-oriented vehicle model for trajectory tracking}

In order to design effective trajectory tracking controller, it is necessary to formulate the longitudinal and lateral dynamics of the vehicle. For modeling the lateral vehicle dynamics, the two wheeled bicycle model is used (Fig. 1.)

Using the notations

$$
\alpha_{1}=\left(\delta-\beta-\dot{\psi} l_{1} / \dot{\xi}\right) \text { and } \alpha_{2}=\left(-\beta+\dot{\psi} l_{2} / \dot{\xi}\right)
$$

the motion equations for lateral and longitudinal dynamics are as follows:

$$
\begin{aligned}
& J \ddot{\psi}=c_{1} l_{1} \alpha_{1}-c_{2} l_{2} \alpha_{2}+M_{b r} \\
& m \dot{\xi}(\dot{\psi}+\dot{\beta})=c_{1} \alpha_{1}+c_{2} \alpha_{2} \\
& m \ddot{\xi}=F_{l}-F_{d} \\
& \ddot{y}_{v}=\dot{\xi}(\dot{\psi}+\dot{\beta})
\end{aligned}
$$

where $m$ is the mass, $J$ is the yaw-inertia of the vehicle, $l_{1}$ and $l_{2}$ are geometric parameters, $c_{1}$ and $c_{2}$ are cornering stiffness which can be identified (Song et al., 2002). The yaw of the vehicle is denoted with $\psi$, while $\beta$ is the side-slip angle, $y_{v}$ is the lateral and $\xi$ is the longitudinal displacement. The nonlinearity of the system is caused by the longitudinal velocity. The actuators of the system are the front steering angle $(\delta)$, the brake yaw moment $\left(M_{b r}\right)$ and the longitudinal force $\left(F_{l}\right)$.

The disturbance force $F_{d}$ affects the longitudinal dynamics of the vehicle. The elements of disturbances are the following: Gravity force disturbance originating from road slope $\alpha: F_{d l}=g \sin \alpha$. Drag disturbance, depending on drag co-efficient $c_{w}$, density of the air $\rho$, contact surface size $A$ and vehicle speed: $F_{d 2}=0.5 c_{w} \rho A \dot{\xi}^{2}$. Rolling resistance depending on vehicle mass $\mathrm{m}$, road surface $\mathrm{K}$ and road slope angle $\alpha: F_{d 3}=m g K \cos \alpha$. In the design of trajectory and velocity tracking it is necessary to guarantee that the longitudinal and lateral positions of the vehicle track the geometry of the road.

The required longitudinal motion is ensured by velocity tracking, while the required lateral motion is controlled by the error between lateral position and the desired road curve. The reference of the road geometry is defined by using a world coordinate system $\left(X_{g l}\right.$ and $\left.Y_{g l}\right)$, in which the coordinate system of the vehicle rotates together with the vehicle. The lateral position of the vehicle is calculated in both coordinate systems, see $y_{v}$ and $y_{g l}$ in Fig. 1. Therefore, it is necessary to consider the rotation of the vehicle in the calculation of the reference road geometry.

\section{Control design based on the LPV method}

The motion equation of the vehicle is transformed into a state-space representation form:

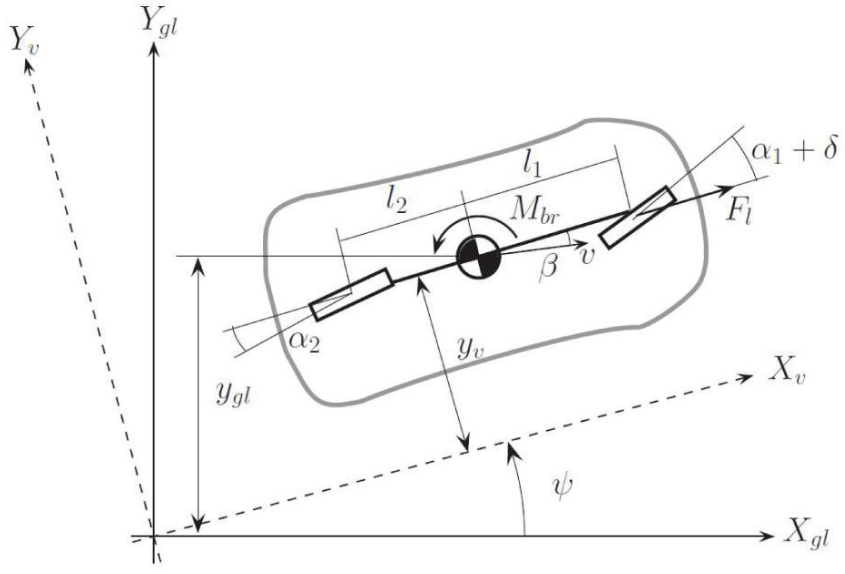

Fig. 1. Single track vehicle model

$$
\begin{aligned}
& \dot{x}=A(\rho) x+B_{1} w+B_{2}(\rho) u \\
& z=C_{1} x+D_{1} w \\
& y=C_{2} x+D_{2} w
\end{aligned}
$$

The state vector of the system:

$$
x=\left[\begin{array}{llllll}
\dot{\xi} & \xi & \dot{\psi} & \beta & \dot{y}_{v} & y_{v}
\end{array}\right]^{T}
$$

contains the longitudinal velocity and the displacement of the vehicle, the yaw-rate, the side-slip angle, the lateral velocity and position. The control inputs of the system are the longitudinal force, the front steering angle and the brake yaw-moment:

$$
u=\left[\begin{array}{lll}
F_{l} & \delta & M_{b r}
\end{array}\right]^{T}
$$

In the trajectory tracking both the longitudinal and lateral dynamics must be taken into consideration, i.e., the vehicle must track two reference signals. First of all it is necessary to ensure appropriate velocity tracking in longitudinal direction: $z_{\xi}=\left|\dot{\xi}_{\text {ref }}-\dot{\xi}\right|$. This requirement is formulated as an optimization criterion: $z_{\xi} \rightarrow 0$. Second, the difference between the lateral position of the vehicle and the reference lateral position $z_{y}=\left|y_{v, r}-y_{v}\right|$ must be minimized: $\mathrm{z}_{\mathrm{y}} \rightarrow 0$. These performances are built in a performance vector:

$$
z_{1}=\left[\begin{array}{ll}
z_{\xi} & z_{y}
\end{array}\right]^{T}
$$

Simultaneously, the actuator saturations must be avoided. The maximal forces of both the driveline and braking systems are determined by their physical construction limits and the tyre-road adhesion conditions too. These limits must be taken into consideration in the control design. Thus, they are formulated as performance criteria:

$$
z_{2}=\left[\begin{array}{lll}
\delta & M_{b r} & F_{l}
\end{array}\right]^{T}
$$


The system matrice depends on the velocity of the vehicle nonlinearly. It is assumed that the velocity is available, i.e., it is measured or estimated, see e.g (Scacchioli et al., 2009). Using a scheduling vector $\rho$ with the scheduling variable $\rho=\dot{\xi}$ the nonlinear model is transformed into an LPV model. The measured output of the system is the velocity and the lateral position, i.e., $y=\left[\begin{array}{ll}\dot{\xi} & y_{v}\end{array}\right]^{T}$. For the measurement of the lateral position, differential GPS data, magnetometers or in-vehicle camera systems are used (Rajamani).

The control design is based on a weighting strategy, which is formulated through a closed-loop interconnection structure, see in Fig. 2. Input and output weighting functions are typically selected to the specifications of disturbances and the inverse of the specifications on the outputs. Usually the purpose of weighting function $W_{p}$ is to define the performance specifications in such a way that a trade-off is guaranteed between them. They can be considered as penalty functions, i.e. weights should be large where small signals are desired and small where large performance outputs can be tolerated. The purpose of the weighting functions $W_{w}$ and $W_{n}$ is to reflect the disturbance and sensor noises. $\Delta$ block contains the uncertainties of the system, such as unmodelled dynamics and parameter uncertainty. The magnitude of the neglected dynamics is handled by a weighting $W_{u}$.

In the trajectory tracking problem two performance signals are introduced in order to guarantee the tracking of the reference velocity and lateral displacement with an acceptably small error. The weighting function is selected as a second-order proportional form:

$$
W_{p}=\lambda\left(\alpha_{2} s^{2}+\alpha_{1} s+1\right) /\left(T_{1} s^{2}+T_{2} s+1\right)
$$

Where $\lambda, \alpha_{1,2}$ and $T_{1,2}$ are designed parameters. Similarly weighting functions $W_{u}, W_{w}$ and $W_{n}$ are also selected in a linear and proportional form. Note that although weighting functions are formulated in the frequency domain, their state-space representation forms are applied in the weighting strategy and in the control design.

Three weighting functions are applied for the proper intervention of the actuators (see $W_{a c t}$ in Fig. 2). The brake yawmoment is represented with weighting function $W_{\text {brake }}$, the steering angle with $W_{\text {steer }}$ and a the longitudinal force with $W_{\text {force }}$. The proper design of these weighting functions are crucial, in order to avoid saturation of the propulsion, the brake or the steering system and to ensure a good balance between the actuators. The weighting functions of the steering $W_{\text {steer }}$ and differential braking $W_{\text {brake }}$ are selected in similar forms as $W_{p}$ in (12). The tuning parameters of the above described weighting functions and the parameters of the system model are specified later in Section 5.

The control design is based on the LPV method that uses parameter-dependent Lyapunov functions, see (Bokor et al., Wu et al.). The quadratic LPV performance problem is to choose the parameter-varying controller in such a way that the resulting closed-loop system is quadratically stable and the induced $L_{2}$ norm from the disturbance to the performances is less than the value $\gamma$.

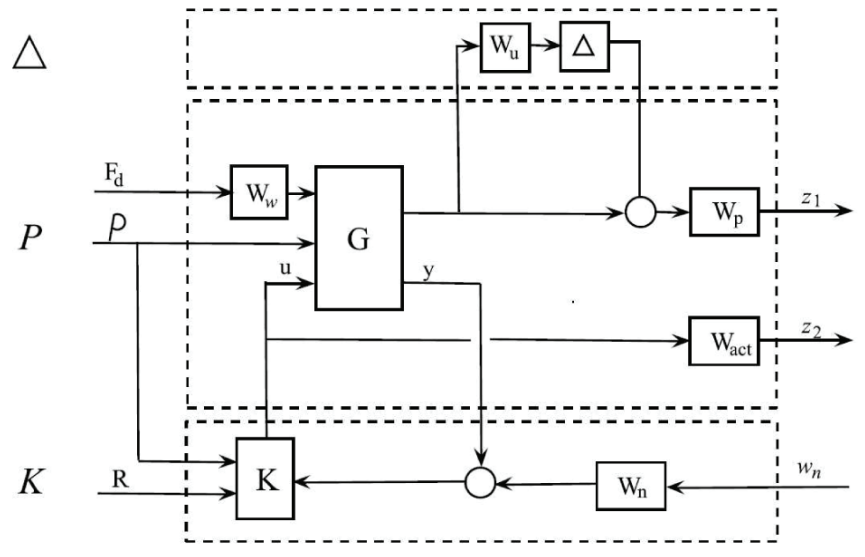

Fig. 2. Closed-loop interconnection structure

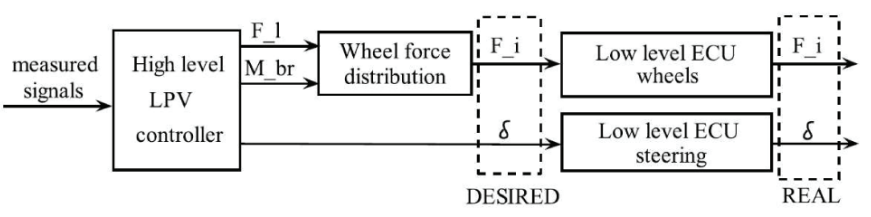

Fig. 3. Architecture of control system

The existence of a controller that solves the quadratic LPV $\gamma$-performance problem can be expressed as the feasibility of a set of Linear Matrix Inequalities (LMIs), which can be solved numerically. Finally, the state space representation of the LPV control $\mathrm{K}(\rho)$ is constructed, see (Wu et al.).

\section{Control implementation}

For the implementation of the control system a hierarchical structure is designed. The synthesis of the multi-layer control system is illustrated in Figure 3.

The purpose of this first layer is to calculate the control inputs, i.e. to define the necessary longitudinal control force, the front steering angle and the brake yaw moment. The function of the second layer is to distribute the input signals designed by the high level controller.

Since the front steering angle is determined factually by the first layer, only construction limits of the steering system is considered, i.e the steering angle is limited by $\delta_{\text {min }} \leq \delta \leq \delta_{\max }$. Thus the main task of the second layer is to distribute the designed longitudinal force and brake yaw moment between the four wheels of the vehicle.

When the vehicle accelerates the distribution of wheel forces depends on the construction of the driving system. In this paper a four wheel driven vehicle is used. Here the propulsion force is distributed equally between all four inwheel motors. The maximal positive and negative forces are determined by physical construction limits of the in-wheel motors and the brake system as well as the tire-road adhesion conditions too. 
The wheel force distribution strategy in the braking process is basically different because of the construction of the brake system. At deceleration the load of wheels are modified because of the pitch dynamics of the vehicle. The brake of the front wheels must be stronger while the brake of the rear wheels must be reduced. The optimal brake force distribution can be determined according to the following form, see (Zomotor):

$$
\begin{aligned}
& F_{r}=-F_{f r}-\left(m g l_{2}\right) /(2 h)+ \\
& +\sqrt{\left(F_{f r}\left(l_{1}+l_{2}\right) m g / h\right)+\left(m g l_{2} / 2 h\right)^{2}}
\end{aligned}
$$

where $F_{r}$ and $F_{f r}$ are the wheel forces at the rear and at the front, $h$ is the height of the central of gravity of the vehicle, respectively.

The brake moment applied on the vehicle by differential braking of the rear wheels $M_{b r}$ is constrained by the principle of limiting the friction. This maximum moment can be calculated with the following equation:

$$
M_{b r \max }=-M_{b r \min }=\mu F_{z r} d
$$

where $\mu$ is the friction coefficient, $d$ is the half length of the rear axle (see Fig. 4). The normal load $F_{z r}$ of the rear axle is given by:

$$
F_{z r}=m g l_{1} /\left(l_{1}+l_{2}\right)
$$

The moment applied at the vehicle center is given by differential braking of the rear wheel. The relation between this moment and the braking force is given as:

$$
M_{b r}=\left(F_{x x, R}-F_{x r, L}\right) d
$$

where $F_{x r, L}$ and $F_{x r, R}$ are the longitudinal forces of the rear wheels. These limits of the actuators must be taken into

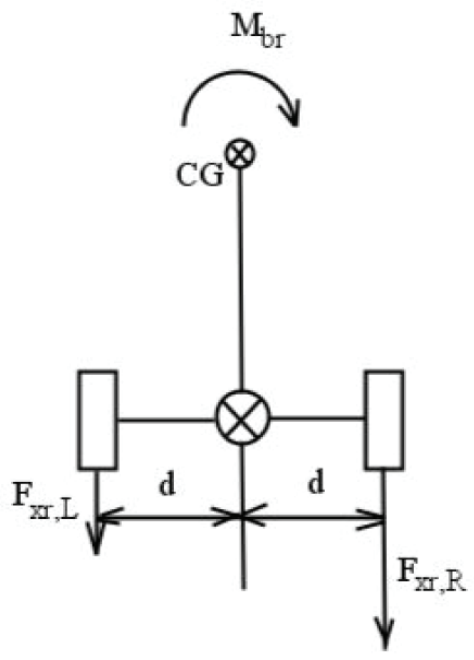

Fig. 4. Rear axle geometry consideration in the control design. Note, that when differential braking is applied in a braking situation, the corresponding rear wheel forces $F_{x r, L}$ and $F_{x r, R}$ are added to the braking force calculated with (13) with respect to the constraint given by (14) .

A third layer is also necessary since the required control forces must be tracked by using low-level controllers. These controllers transform the wheel forces and the values of the steering angle into a real physical parameter of the actuator. The design of lowlevel controllers might use more specific techniques that fit the specific nonlinear properties of the actuator. The steer-by-wire front steering system transforms the steering angle into a real physical parameter of the actuator. The real physical input of the system is the Pulse Width Modulated (PWM) signal of the electric servo motor, which moves the rack. The physical construction of electric steering has a lot of variations, see e.g. (Kozaki et al.).

\section{Simulation results}

The vehicle chosen for simulation is driven by all four wheels using in-wheel electric motors, while the braking is performed with hydraulic brake system. The parameters of the vehicle are shown in Table 1.

The geometry of the racetrack is shown in Fig. 5. As it can be seen, the path of the vehicle contains curves and hilly roads as well, thus to track a desired velocity and path at the same time can be difficult and somewhat impossible without the optimal coordination of the steering, braking and propulsion system of the vehicle.

The X-Y plane of the racetrack is shown in Fig. 5(a) along with the terrain characteristics in Fig. 5(b). The target velocity for the vehicle to follow is shown in Fig. 5(c). The start line of the racetrack is at the zero value of $\mathrm{x}$ and $\mathrm{y}$ coordinates. The vehicle has to perform a full lap on the racetrack with the changing target velocity without leaving the trajectory defined by the racetrack. In the following two different weighting strategies will be examined.

The performance weighting $W_{p}$ is divided into the weighting of the lateral position error $W_{\text {position }}$ and the weighting of the velocity error $W_{\text {velocity }}$. The parameters of $W_{\text {position }}$ are the following: $\lambda_{\mathrm{p}}=20000, \alpha_{\mathrm{p} 1}=1 / 7, \alpha_{\mathrm{p} 2}=1 / 200, T_{\mathrm{p} 2}=2 / 10$ and $T_{\mathrm{p} 1}=1 / 100$. The parameters of $W_{\text {velocity }}$ are the following: $\lambda_{\mathrm{v}}=833, \alpha_{\mathrm{v} 1}=1 / 100, \alpha_{\mathrm{v} 2}=0, T_{\mathrm{v} 2}=2 / 10$ and $T_{\mathrm{v} 1}=0$.

The actuators are weighted as follows. For the steering weighting function the following parameters are chosen: $\lambda_{\mathrm{s}}=1 / 100, \alpha_{\mathrm{s} 1}=2 / 100, \alpha_{\mathrm{s} 2}=1 / 10000, T_{\mathrm{s} 2}=2 / 10$ and $T_{\mathrm{s} 1}=1 / 100$. The braking weighting function parameters are the following: $\lambda_{\mathrm{b}}=5 / 100000, \alpha_{\mathrm{b} 1}=2 / 10, \alpha_{\mathrm{b} 2}=1 / 100, T_{\mathrm{b} 2}=2 / 100$ and $T_{\mathrm{b} 1}=1 / 10000$. The longitudinal force is weighted with $W_{\text {force }}=1$.

The unmodeled dynamics are weighted with $W_{u}$ as follows: $\lambda_{\mathrm{u}}=1 / 100000, \alpha_{\mathrm{u} 1}=2 / 10, \alpha_{\mathrm{u} 2}=1 / 100, T_{\mathrm{u} 2}=4 / 1000$ and $T_{\mathrm{u} 1}=4 / 1000000$. The sensor noise weight is $W_{n}=1 / 1000$, the disturbance weight is $W_{w}=1 / 2$.

The controller designed with the above weighting selection enables the vehicle to operate without saturation of the 
Tab. 1. Vehicle parameters

\begin{tabular}{ccc}
\hline Parameter & Value & Unit \\
\hline $\mathrm{m}$ & 830 & $\mathrm{~kg}$ \\
$\mathrm{~J}$ & 1110.9 & $\mathrm{kgm} 2$ \\
$\mathrm{I}_{1}$ & 1.103 & $\mathrm{~m}$ \\
$\mathrm{I}_{2}$ & 1.244 & $\mathrm{~m}$ \\
$\mathrm{~b}_{\mathrm{f}}$ & 1.416 & $\mathrm{~m}$ \\
$\mathrm{~b}_{\mathrm{r}}$ & 1.375 & $\mathrm{~m}$ \\
$\mathrm{c}_{1}$ & 22000 & $\mathrm{~N} / \mathrm{rad}$ \\
$\mathrm{c}_{2}$ & 85000 & $\mathrm{~N} / \mathrm{rad}$ \\
$\mathrm{c}_{\mathrm{w}}$ & 0.343 & \\
$\mathrm{~A}$ & 1.6 & $\mathrm{~m} 2$ \\
\hline
\end{tabular}

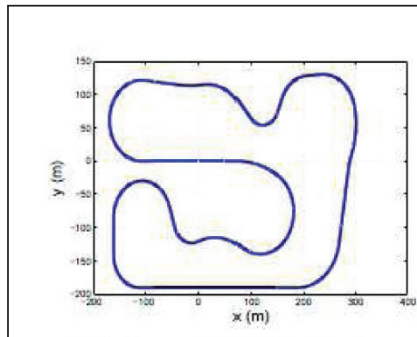

(a) Racetrack X-Y plane

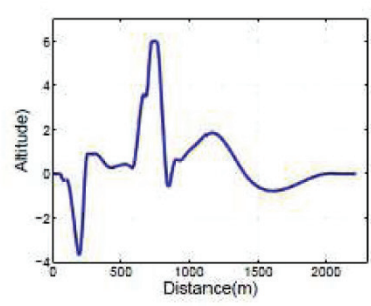

(b) Altitude

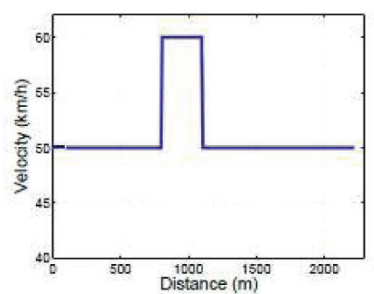

(c) Reference velocity

Fig. 5. Reference velocity and trajectory

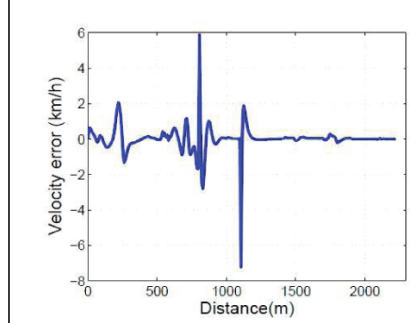

(a) Velocity error of vehicle

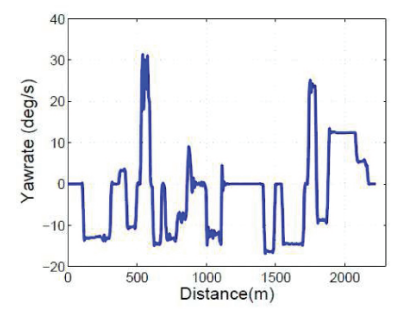

(c) Yawrate

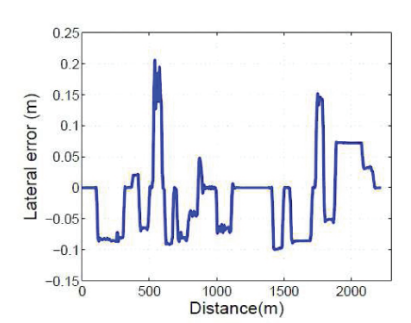

(b) Lateral error of vehicle

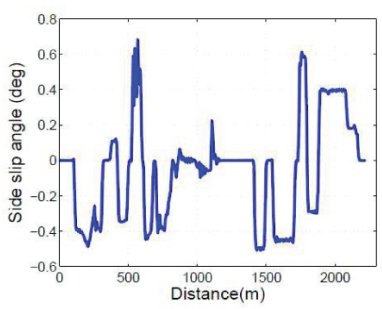

(d) Side slip angle
Fig. 7. Time responses in Case 1 actuators and achieves trajectory tracking with the dominance of the steering over differential braking. The Bode plot of the designed weighting functions are shown in Fig. 6.

It is well demonstrated in Fig. 7(a) and 7(b) that the designed controller tracks well both the reference velocity and trajectory with acceptable errors from the reference values. The lateral error does not exceed $0.2 \mathrm{~m}$ even in the sharpest corner of the racetrack. Thus, the vehicle follows the trace of the racetrack with a predefined velocity profile without the danger of skidding or rollover. The yaw rate and side slip angle of the vehicle can be seen in Fig. 7(c) and 7(d). It can be observed, that during heavy cornering both yaw rate and side slip angle can increase significantly.

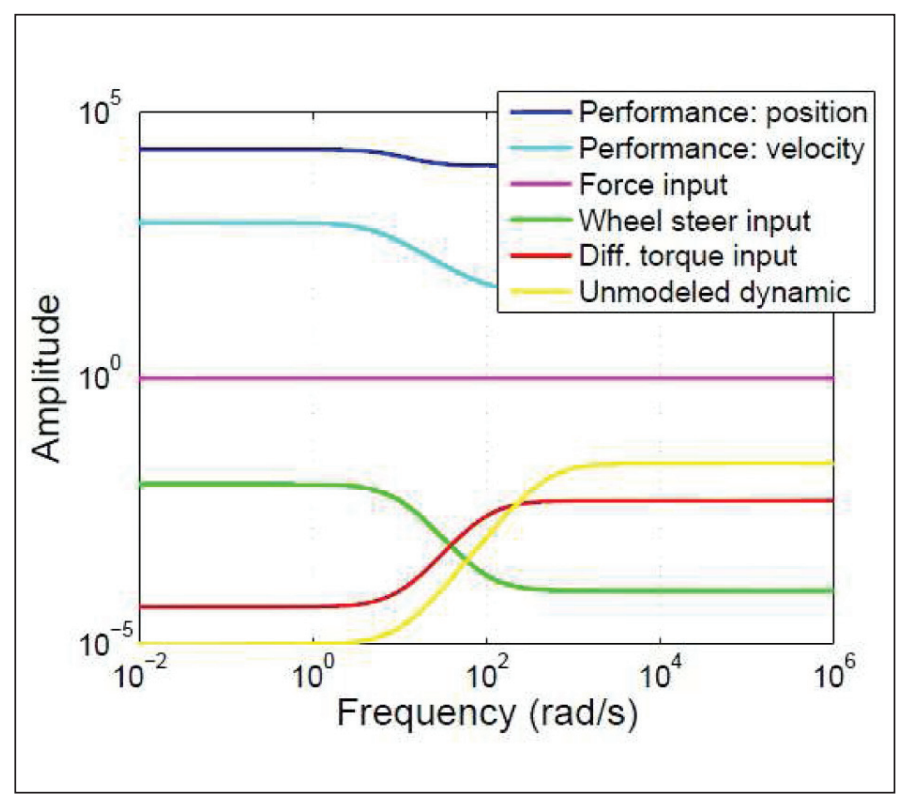

Fig. 6. Bode plot of the weighting functions in Case 1
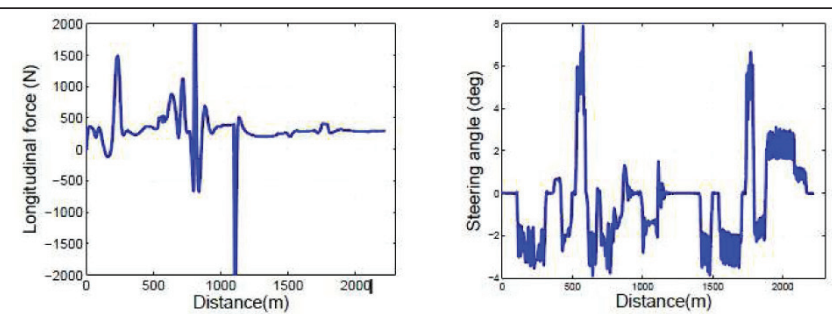

(a) Actuated longitudinal (b) Actuated steering angle force

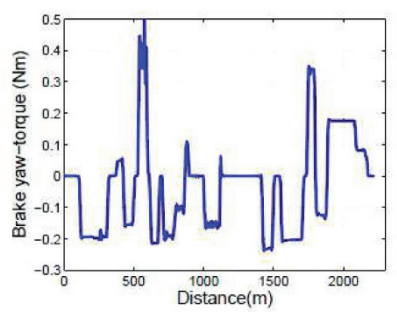

(c) Actuated brake yawmoment

Fig. 8. Control signals in Case 1 


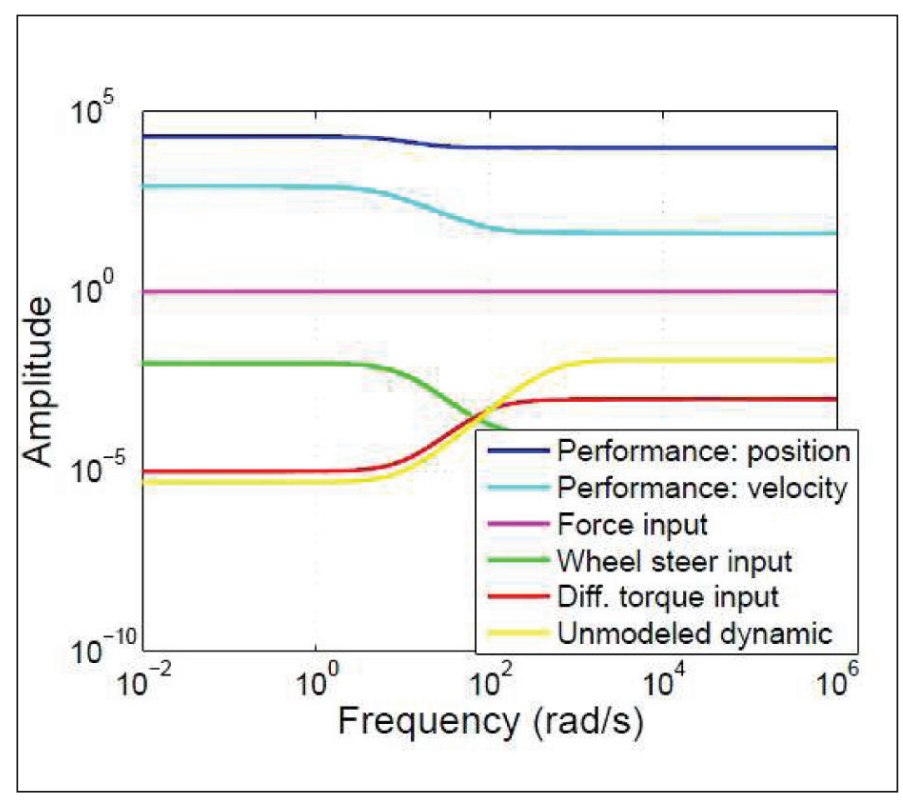

Fig. 9. Bode plot of the weighting functions in Case 2

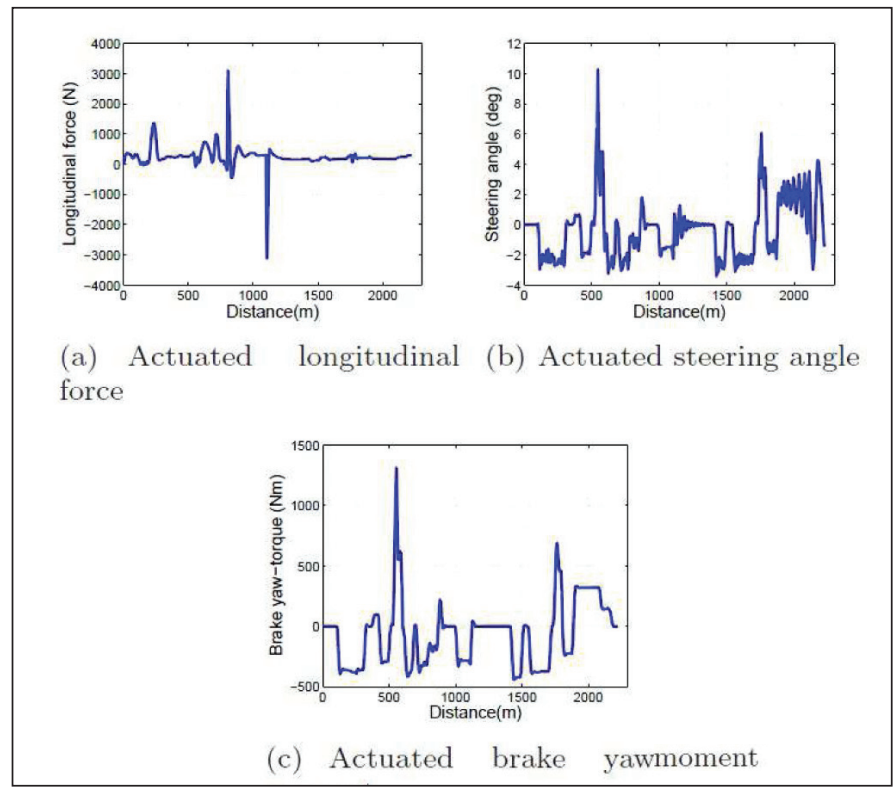

Fig. 11. Control signals in Case 2

The motion of the vehicle is influenced by the inputs of the designed controller. These are the longitudinal force distributed between the four wheels, the steering angle acting on the front wheels and the brake yaw-moment generated by differential braking of the rear wheels. The actuated longitudinal force varies depending on the task of braking or accelerating, see Fig. 8(a). Heavy acceleration and deceleration corresponding with the changing speed limit can also be observed. Fig. 8(b) shows the steering angle of the vehicle, which is the dominant control signal for the task of trajectory tracking. The actuated brake yaw-moment is moderate due to the steering focused weighting strategy of the high level controller, see Fig. 8(c).

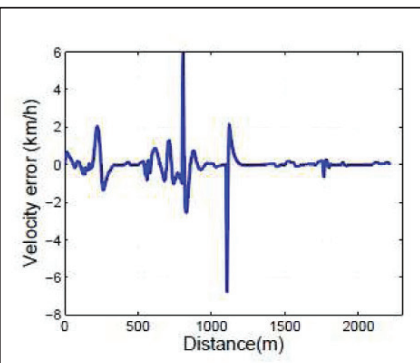

(a) Velocity error of vehicle

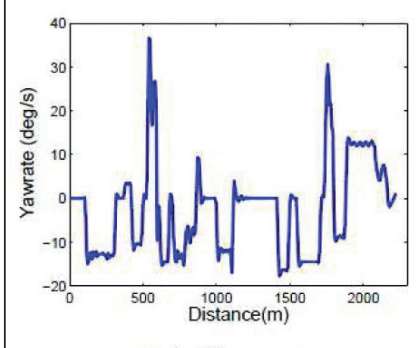

Fig. 10. Time responses in Case 2

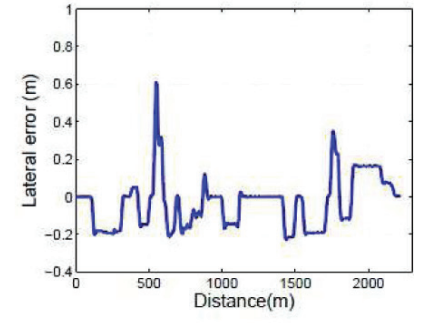

(b) Lateral error of vehicle

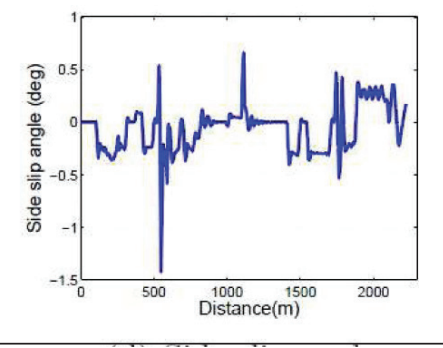

In order to the illustrate the role of the weighting functions, the design of the high level LPV controller is modified by changing the weighting function of the differential braking with the following parameters: $\lambda_{b}=1 / 100000, \alpha_{b 1}=2 / 10$, $\alpha_{\mathrm{b} 2}=1 / 100, T_{\mathrm{b} 2}=2 / 100$. The modified differential braking weighting function can be observed in Fig. 9. With the selected weighting functions the amount of differential braking is significant compared to the previous case.

The simulation results performed with the modified high level controller are shown in Fig. 10 and Fig. 11. It is well demonstrated, that the implemented yaw torque of the vehicle is increased compared to the first case, see Fig. 8(c) and Fig. 11(c). With the modified weights the steering is generally moderated (see Fig. 11(b)), thus the cornering of the vehicle is realized by the intervention of differential braking and the steering as well.

\section{Conclusion}

The paper has proposed the design of an integrated vehicle control system for in-wheel electric vehicle, which is able to track road geometry with a predefined reference velocity. In the design the lateral and longitudinal dynamics are combined using the propulsion, the brake and the steering system of the vehicle as actuators. The control design is based on LPV method considering performance specifications, model uncertainties and disturbances. 


\section{Acknowledgements}

The project has been supported by the Foundation for Hungarian Transportation Engineering and the HungaroControl, the Hungarian Air Navigation Services Co. The research has also been conducted as part of the project TÁMOP-4.2.2.A-11/1/KONV-2012-0012: Basic research for the development of hybrid and electric vehicles.

\section{References}

1 Anwar S., Zheng B., Yaw stability control of a steer-by-wire equipped vehicle via active front wheel steering. Mechatronics, 19(6), 799-804 (2009).

DOI: 10.1016/j.mechatronics.2009.04.005

2 Baslamisli S., C., Polat I., Kose I. E., (2008). Gain sched-uled integrated vehicle control based on a parametricyaw roll model. in '2008 IEEE Intelligent Vehicles Symposium. Eindhoven, Netherlands', 666-671 (2008). DOI: 10.1109/IVS.2008.4621163

3 Bokor J., Balas G., Linear parameter varying systems: A geometric theory and applications. in '16th IFAC World Congress. Prague, Czech Republic', 16(1), (2005). DOI: 10.3182/20050703-6-CZ-1902.00003

4 Dugard L., Martinez-Molina J., Gáspár P., Szabó Z., Doumiati M., Sename O., Integrated vehicle dynamics control via coordination of active front steering and rear braking. European Journal of Control, 19(2), 121-143 (2013).

DOI: $10.1016 /$ j.ejcon.2013.03.004

5 Gáspár P., Szabó Z., Bokor J., Tracking control by integrated steering and braking systems using an observer-based estimation. in 'Proceedings of the European Control Conference, ECC'07. Kos, Greece', (2007).

6 Hori Y., Kawashima K., Uchida T., Rolling stability control of in-wheel electric vehicle based on two-degree- of-freedom control. in 'Proceeding of Advanced Motion Control, 10th IEEE International Workshop. Trento, Italy', 751-756 (2008). DOI: 10.1109/AMC.2008.4516161

7 Jianyong W., Houjun T., Shaoyuan L., Wan F., Improvement of vehicle handling and stability by integrated control of four wheel steering and direct yaw moment. in 'Proceeding 26th Chinese Control Conference. Zhangjiajie, Hunan, China', 730-735 (2007). DOI: $\underline{10.1109 / C H I C C .2006 .4347270}$

$8 \mathrm{Kim} \mathrm{H}-\mathrm{J} .$, Robust roll motion control of a vehicle using integrated control strategy. Control Engineering Practice, 19(8), 820-827 (2011). DOI: 10.1016/j.conengprac.2011.04.009

9 Kozaki Y., Hirose G., Sekiya S., Miyaura Y., Electric power steering (EPS). Motion and Control, 6, 9-15 (1999).

10 Mastinu G., Babbel E., Lugner P., Margolis D., Integrated controls of lateral vehicle dynamics. Vehicle System Dynamics, 23(1), 358-377 (1994). DOI: $\underline{10.1080 / 00423119308969527}$ 